\title{
Equilibrium statistics of a slave estimator in Langevin processes
}

\author{
David S. Dean ${ }^{(1,2)}$, Ian T. Drummond ${ }^{(1)}$, Ron R. Horgan ${ }^{(1)}$ and Satya N. Majumdar ${ }^{(2,3)}$ \\ (1) DAMTP, CMS, University of Cambridge, Cambridge, CB3 OWA, UK \\ (2) Laboratoire de Physique Théorique, UMR CNRS 5152, IRSAMC, \\ Université Paul Sabatier, 118 route de Narbonne, 31062 Toulouse Cedex 04, France \\ (3) Laboratoire de Physique Théorique et Modèles Statistiques, \\ UMR 8626, Université Paris Sud, Bât 100, 91045 Orsay Cedex, France
}

(Dated: 21st September 2004)

\begin{abstract}
We analyze the statistics of an estimator, denoted by $\xi_{t}$ and referred to as the slave, for the equilibrium susceptibility of a one dimensional Langevin process $x_{t}$ in a potential $\phi(x)$. The susceptibility can be measured by evolving the slave equation in conjunction with the original Langevin process. This procedure yields a direct estimate of the susceptibility and avoids the need, when performing numerical simulations, to include applied external fields explicitly. The success of the method however depends on the statistical properties of the slave estimator. The joint probability density function for $x_{t}$ and $\xi_{t}$ is analyzed. In the case where the potential of the system has a concave component the probability density function of the slave acquires a power law tail characterized by a temperature dependent exponent. Thus we show that while the average value of the slave, in the equilibrium state, is always finite and given by the fluctuation dissipation relation, higher moments and indeed the variance may show divergences. The behavior of the power law exponent is analyzed in a general context and it is calculated explicitly in some specific examples. Our results are confirmed by numerical simulations and we discuss possible measurement discrepancies in the fluctuation dissipation relation which could arise due to this behavior.
\end{abstract}

\section{INTRODUCTION}

A standard experimental technique for probing a system is to measure its response to a small external field. In equilibrium, static response functions are related through the fluctuation dissipation relation to appropriate static correlation functions. A way to measure such responses in the context of numerical simulations is the slave equation method which is used for Langevin type systems, and more precisely in the context of stochastic quantization [1]. Via the static fluctuation dissipation theorem the slave equation method can be used to compute correlation functions and has been successfully exploited in numerical simulations of quantum field theories and statistical spin systems [2, 3]. The advantage of the slave equation method is that it provides a way of measuring cumulant correlators directly with properly estimated statistical errors. As has been noted however [2, 3], there are circumstances in which the slave equation method breaks down. These difficulties are clearly illustrated by the simple model investigated in this paper. Another advantage of the slave method is that the response can be measured without imposing a small external field and thus unperturbed correlation functions can be measured simultaneously. Recently various numerical methods for measuring response functions in discrete spin systems, without applying an external field, have been proposed [4].

Although not the subject of this paper, it is worth noting that for systems in equilibrium more general fluctuation dissipation theorems exist that relate dynamical response functions and dynamical correlation functions. In out of equilibrium systems that exhibit aging various types of fluctuation dissipation theorems have been to shown to hold in mean field models and numerical and experimental evidence points to their validity in finite dimensional systems 5]. Forms of fluctuation dissipation relations/theorems are also expected to hold in the steady state of certain non-equilibrium driven systems.

The model we investigate is the simplest possible, namely an over-damped particle moving in one dimension in a background potential $\phi(x)$ subject to an external field $h$ and thermal noise. The Langevin equation for this system is

$$
\dot{x}_{t}=-\phi^{\prime}\left(x_{t}\right)+h+\eta_{t}
$$

where $\eta_{t}$ is zero mean Gaussian white noise with correlation function

$$
\left\langle\eta_{t} \eta_{t^{\prime}}\right\rangle=2 T \delta\left(t-t^{\prime}\right)
$$

with $T$ the temperature. At zero external field we define the response function $\xi$ by

$$
\xi_{t}=\left.\frac{\partial x_{t}}{\partial h}\right|_{h=0}
$$


Differentiating Eq.(1) with respect to $h$ and setting $h=0$ we obtain the equation of motion of $\xi_{t}$ as

$$
\dot{\xi}_{t}=-\phi^{\prime \prime}\left(x_{t}\right) \xi_{t}+1 \text {. }
$$

The variable $\xi_{t}$ is referred to as the slave and Eq.(4) the slave equation corresponding to the process $x_{t}$. This terminology is obvious upon examining Eqs.(11) and (44) as we see that the process $\xi_{t}$ is driven by the process $x_{t}$ but the evolution of $x_{t}$ is completely independent of $\xi_{t}$. Of course the analysis can be generalized to higher dimensions. This is separately interesting and will be addressed in future work.

Processes whose evolution is similar to that given by Eq. (4) arise in a variety of physical contexts such as the development of curvature in material line and surface elements and magnetic fields transported by random flows [6, 7, [8, 9]. Indeed the behavior exhibited by the probability density functions of the relevant slave variables is exactly as analyzed in our simple model. A related slave variable $\zeta_{t}$,

$$
\zeta_{t}=\frac{\partial x_{t}}{\partial x_{0}}
$$

where $x_{0}$ is the initial value of $x_{t}$, satisfies the slave equation

$$
\dot{\zeta}_{t}=-\phi^{\prime \prime}\left(x_{t}\right) \zeta_{t}
$$

Eq. (6) is identical to Eq. (44) except for the inhomogeneous term +1 on the right. Although the two equations are close in form the presence of this inhomogeneous term radically affects the statistics of $\xi_{t}$. The behavior of the slave variable $\zeta_{t}$ is is a measure of the sensitivity of a system to its initial conditions and thus related to Lyapounov exponents. It was recently shown how Lyapounov exponents could be analyzed via supersymmetric quantum mechanics [10], our approach is not explicitly developed in terms of supersymmetry but it is clear that it could be rewritten in these terms.

In equilibrium in the presence of an external field $h$, the statistics of the process $x_{t}$ is described by the GibbsBoltzmann distribution

$$
P_{G B}(x)=\frac{1}{Z(h)} \exp (-\beta \phi(x)+\beta h x)
$$

where

$$
Z(h)=\int d x \exp (-\beta \phi(x)+\beta h x)
$$

is the canonical partition function for the process $x$. Clearly one has, by linearity of an expectation of a probability distribution, that

$$
\langle\xi\rangle_{E}=\frac{\partial}{\partial h}\langle x\rangle_{E}
$$

where the subscript $E$ denotes expectations taken in the equilibrium state. Using the form of the Gibbs-Boltzmann distribution then yields the static fluctuation-dissipation theorem

$$
\langle\xi\rangle_{E}=\beta\left(\left\langle x^{2}\right\rangle_{E}-\langle x\rangle_{E}^{2}\right)
$$

In simulations, the mean of $\xi$ in equilibrium is easy to determine. It therefore provides, up to a multiplicative factor, a direct estimator for the variance of $x$. The numerical usefulness of this result is two-fold. First a straight calculation of the variance computed as in Eq.(10) is not ideal from the the point of view of precision, as it involves the subtraction of two numbers, each potentially much larger than their difference. Measuring $\xi$ avoids this difficulty. Second, from the variance of $\xi$ we have a proper statistical estimate for the error in the susceptibility and the variance of $x$. The utility of the slave method hinges precisely on the existence and size of the variance of $\xi$, the slave variable.

Given the importance of $\xi$ as an estimator to the variance of $x$ by the fluctuation-dissipation relation and its direct physical significance as a susceptibility, it is natural to investigate its statistical properties and in particular its equilibrium distribution function which we shall denote by $\rho(\xi)$. In this paper, we address this issue in detail.

The equilibrium probabilty density of $\xi, \rho(\xi)$, may be obtained from the joint probability density function (PDF) $P(x, \xi)$ via ,

$$
\rho(\xi)=\int d x P(x, \xi)
$$


The joint PDF satisfies [13], in equilibrium

$$
-H_{F P} P(x, \xi)+\frac{\partial}{\partial \xi}\left(\left(\phi^{\prime \prime}(x) \xi-1\right) P(x, \xi)\right)=0,
$$

where $H_{F P}$ is the forward Fokker Planck operator for the process $x_{t}$ and is defined by

$$
H_{F P} P=-T \frac{\partial^{2}}{\partial x^{2}} P-\phi^{\prime}(x) \frac{\partial}{\partial x} P-\phi^{\prime \prime}(x) P \text {. }
$$

The Gibbs-Boltzmann distribution for $x, P_{G B}(x)$ of Eq. (7), is recovered via

$$
P_{G B}(x)=\int d \xi P(x, \xi)
$$

and of course satisfies

$$
-H_{F P} P_{G B}(x)=0
$$

\section{STATIC FLUCTUATION DISSIPATION RELATION}

The fundamental nature of the static fluctuation dissipation relation between the equilibrium susceptibility and the systems variance means it is illuminating to verify the relation Eq.(10) from Eq.(12 directly). We set

$$
P(x, \xi)=P_{G B}(x) F(x, \xi),
$$

and define $F_{n}(x)$, where it exists, by the equation

$$
F_{n}(x)=\int d \xi \xi^{n} F(x, \xi)
$$

Clearly

$$
F_{0}(x)=1
$$

and

$$
\langle\xi\rangle_{E}=\int d x P_{G B}(x) F_{1}(x)
$$

It follows from Eq. (12) that

$$
\left(T \frac{\partial}{\partial x}-\phi^{\prime}(x)\right) \frac{\partial}{\partial x} F(x, \xi)+\frac{\partial}{\partial \xi}\left(\left(\phi^{\prime \prime}(x) \xi-1\right) F(x, \xi)\right)=0 .
$$

If we multiply by $\xi$ and integrate over all $\xi$ we find

$$
\left(T \frac{\partial}{\partial x}-\phi^{\prime}(x)\right) \frac{\partial}{\partial x} F_{1}(x)-\phi^{\prime \prime}(x) F_{1}(x)+1=0 .
$$

This can be rewritten in the form

$$
\frac{\partial}{\partial x}\left(T \frac{\partial}{\partial x} F_{1}(x)-\phi^{\prime}(x) F_{1}(x)+x\right)=0
$$

which implies that

$$
T \frac{\partial}{\partial x} F_{1}(x)-\phi^{\prime}(x) F_{1}(x)+x-a=0,
$$

where $a$ is an integration constant. If we then multiply by $P_{G B}(x)$ and integrate over all $x$ we find

$$
a=\langle x\rangle_{E} .
$$

We now multiply by Eq. (23) by $(x-a) P_{G B}(x)$ and integrate over all $x$ to obtain

$$
-T \int d x P_{G B}(x) F_{1}(x)+\int d x P_{G B}(x)(x-a)^{2}=0,
$$

which is precisely the static fluctuation dissipation relation Eq. (10). The derivation therefore confirms the properties of the estimator $\xi_{t}$ as deduced from the stochastic differential equation for $x_{t}$. 


\section{GENERAL PROPERTIES OF THE $\xi$-PROBABILITY DISTRIBUTION}

It follows from Eq. (44) that for $\xi \simeq 0, \dot{\xi}_{t} \simeq 1$. This implies that there is always a positive flow of probability from negative to positive $\xi$. In equilibrium therefore the support for $P(x, \xi)$ lies in range $\xi>0$. We shall assume also, which can be justified subsequently, that $P(x, 0)=0$.

The Laplace transform of the joint probability function is

$$
\tilde{P}(x, s)=\int_{0}^{\infty} d \xi e^{-s \xi} P(x, \xi)
$$

It follows from our discussion that we expect $\tilde{P}(x, s) \rightarrow 0$ faster than $s^{-1}$ as $s \rightarrow \infty$, and

$$
\int_{0}^{\infty} d \xi e^{-s \xi} \frac{\partial}{\partial \xi} P(x, \xi)=s \tilde{P}(x, s)
$$

The large $\xi$ behavior of $P(x, \xi)$ is also of importance. As will become clear later this is strongly influenced by the behavior of $\phi^{\prime \prime}(x)$. Let there be an interval $U$ such that for $x \in U, \phi^{\prime \prime}(x) \simeq-g$ where $g>0$. It follows from Eq. (4) while $x$ remains in $U$ then $\xi$ will grow exponentially. If the time for which $x$ remains in $U$ is $\tau$ then the excursion experienced by $\xi$ will be roughly of the form

$$
\xi=\xi_{0} e^{g \tau}
$$

for some $\xi_{0}$. When $x$ leaves $U, \xi$ will decay rapidly back to small values. Since the the process $x_{t}$ is essentially without memory the distribution of $\tau$ will be exponential.

$$
p(\tau) \simeq \mu \exp (-\mu \tau) \quad .
$$

The large $\xi$ behavior of $\rho(\xi)$ is determined by these excursions. We have

$$
\rho(\xi) \approx \frac{\mu}{g \xi_{0}}\left(\frac{\xi_{0}}{\xi}\right)^{1+\alpha^{*}},
$$

where $\alpha^{*}=\mu / g$. Hence we can expect a power law in the distribution for large values of $\xi$ when there exists a region $U$ in which the potential $\phi(x)$ is concave. Of course the argument above does not allow an easy calculation or even estimation of the exponent since the time spent in concave regions is affected by the global structure of the potential.

A potential which exhibits a region of concavity and for which therefore we expect to find a power law behavior for $\rho(\xi)$, is $\phi(x)=\left(1-x^{2}\right)^{2} / 4$. We have simulated the Langevin process $x_{t}$ and its slave by integrating Eqs. (1) and (44) using a second order stochastic Runga-Kutta method [12]. Shown in Fig. (11) is the time series obtained for $\xi$ at $\beta=1 / T=1$. The mean value of the process as predicted by the static fluctuation dissipation relation Eq.(10) is shown by the thick horizontal line. A direct measurement of the time series average confirms this result, as it should.

The most striking feature of the time series however is that $\xi_{t}$ spends most of the time below the average value with intermittent spikes rising to large values. These spikes are the means by which the time series fills the power law tail in the distribution for $\rho(\xi)$ as indicated by the intuitive argument explained above. The implication of this result for simulations is that it is essential to include the intermittent upward excursions if correct estimates are to be obtained for the susceptibility. If therefore one were to measure $\langle\xi\rangle_{E}$ by taking a time series average, then in order to obtain a satisfactory estimate one must ensure that the time interval of the measurement is of a length sufficient to sample the rare but large excursions that represent the power law tail of $\rho(\xi)$. The typical value of $\xi$ that one measures during a simulation is well below the mean value. Thus measuring over too short a time scale, or for other reasons omitting the large excursions will lead to one to an under estimate of $\langle\xi\rangle_{E}$.

Another approach to the fluctuation dissipation theorem would be to be to compute numerically or measure physically the expectation value $\xi_{a}$ of the slave variable $\xi$ and the variance of the the original variable $x$ and use these results to provide an estimate $T_{\text {eff }}$ of the temperature of the system.

$$
T_{\text {eff }}=\frac{\left\langle x^{2}\right\rangle_{E}-\langle x\rangle_{E}^{2}}{\xi_{a}} .
$$

If for the reasons discussed above we under estimate $\xi_{a}$ then our estimate for the temperature will be too high,

$$
T_{e f f} \geq T .
$$




\begin{tabular}{|c|c|}
\hline$T$ & $\alpha^{*}$ (numerics) \\
\hline 0.66 & $2.46(3)$ \\
\hline 0.50 & $1.86(2)$ \\
\hline 0.40 & $1.67(1)$ \\
\hline 0.33 & $1.54(1)$ \\
\hline
\end{tabular}

TABLE I: The exponent $\alpha^{*}$, estimated by straight line fit to the tail of the log-log plot of the numerically generated histogram, as a function of $\beta$ for the potential $\phi(x)=\left(1-x^{2}\right)^{2} / 4$.

It is interesting to note that this result with $T_{\text {eff }} / T \geq 1$ is also seen in aging systems where the dynamical fluctuation dissipation theorem is violated because the system is not in equilibrium [11]. However here the apparent violation is due to an error of measurement in an equilibrium state and hence of somewhat different origin.

The value of the exponent $\alpha^{*}$ appearing in Eq. (30) may be obtained from the numerical simulation by fitting a straight line to the large $\xi$ region of the log-log plot of the numerically generated histogram of $\xi$. We have carried out this procedure for a range of values of the temperature $T$ and the results are shown in table (I) . Most importantly we see that the exponent $\alpha^{*}$ depends continuously on temperature. In addition, two features of the behavior of $\alpha^{*}$ in these numerical results stand out. First, as $T \rightarrow \infty$ then it appears $\alpha^{*} \rightarrow \infty$ (as $\alpha^{*}$ increases an accurate fit of the power law tail's exponent becomes difficult due to the lack of statistical weight in the tail). Second, as $T \rightarrow 0$ the numerics is consistent with $\alpha^{*} \rightarrow 1$. This latter result is particularly significant. At low temperature the PDF for $x$ takes the form

$$
P_{G B}(x)=\frac{1}{2}(\delta(x-1)+\delta(x+1)) .
$$

This implies that $\langle x\rangle_{E}=0$ and $\left\langle x^{2}\right\rangle_{E}=1$. It follows from the static fluctuation dissipation relation that

$$
\langle\xi\rangle_{E} \simeq \frac{1}{T}, \quad \text { as } T \rightarrow 0
$$

For large $\xi$ we have

$$
\rho(\xi) \simeq \frac{C}{\xi^{1+\alpha^{*}}}
$$

for some positive $C$. It follows that

$$
\langle\xi\rangle_{E}=\int^{\infty} d \xi \xi \rho(\xi) \simeq \int^{\infty} d \xi \xi \frac{C}{\xi^{1+\alpha *}} \simeq \frac{C}{\left(\alpha^{*}-1\right)} .
$$

Comparing this result with the static fluctuation dissipation relation we see that at low temperature we should expect $\alpha^{*} \rightarrow 1$ and more specifically $C /\left(\alpha^{*}-1\right) \approx 1 / T$. This is consistent with the numerical results shown in table (III) .

For a given value of $\alpha^{*}$ the existence of a power law tail for $\rho(\xi)$ means that moments $\left\langle\xi^{n}\right\rangle_{E}$ diverge for $n>\alpha^{*}$. At low $T$, where $\alpha^{*}<2$, therefore even the variance of the estimator for the susceptibility has become divergent. At this point it has ceased to be a useful estimator and provides no reasonable estimate for a statistical error on the susceptibility. The slave equation method therefore becomes ineffective under these circumstances. These issues in relation to simulations of quantum field theory and spin models in statistical mechanics have been noted before [2, 3].

\section{GENERAL THEORY}

The above observations on the nature of the PDF of $\xi$ are much clarified and rendered more robust by an understanding of the general theory of the joint PDF. In order to pursue the analysis it is convenient to make a standard transformation that renders $H_{F P}$ into self adjoint form. We define $Q(x, \xi)$ so that

$$
P(x, \xi)=Q(x, \xi) \frac{\exp (-\beta \phi(x) / 2)}{Z^{\frac{1}{2}}} .
$$

It follows that $Q$ obeys

$$
-H_{0} Q(x, \xi)+\frac{\partial}{\partial \xi}\left(\left(\xi \phi^{\prime \prime}(x)-1\right) Q(x, \xi)\right)=0
$$




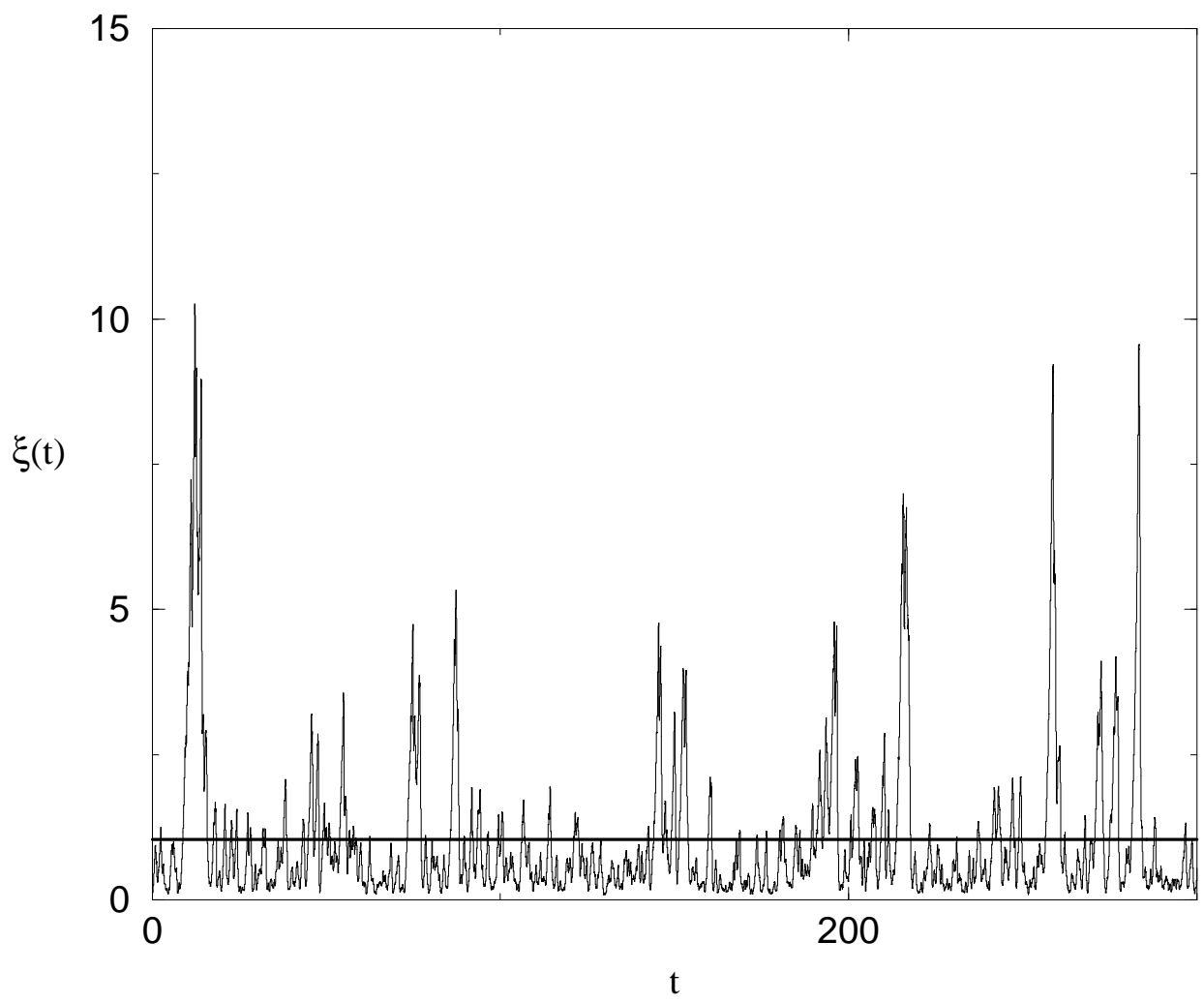

FIG. 1: Time series for slave $\xi_{t}$ with potential $\phi(x)=\left(1-x^{2}\right)^{2} / 4$ at $\beta=1$. Shown by the thick horizontal line is its average value

where the manifestly self adjoint operator $H_{0}$ is given by

$$
H_{0}=-T \frac{\partial^{2}}{\partial x^{2}}+V(x)
$$

with

$$
V(x)=\left(\frac{1}{4 T}\left(\phi^{\prime}(x)\right)^{2}-\frac{1}{2} \phi^{\prime \prime}(x)\right) .
$$

We introduce the Laplace transform of $Q$ with respect to the variable $\xi$

$$
\tilde{Q}(x, s)=\int_{0}^{\infty} d \xi \exp (-s \xi) Q(x, \xi)
$$

and thus corresponding Laplace transform of $P$ is given by

$$
\tilde{P}(x, s)=\frac{\exp (-\beta \phi(x) / 2) \tilde{Q}(x, s)}{Z^{\frac{1}{2}}} .
$$

We note that

$$
\tilde{P}(x, 0)=\frac{\exp (-\beta \phi(x))}{Z}
$$

which thus gives us the initial conditions for $\tilde{Q}$ at $s=0$ to be

$$
\tilde{Q}(x, 0)=\frac{\exp (-\beta \phi(x) / 2)}{Z^{\frac{1}{2}}} .
$$


The evolution equation for $\tilde{Q}$ is

$$
H_{0} \tilde{Q}+s \tilde{Q}+s \frac{\partial}{\partial s} \tilde{Q}=0
$$

We notice that a particular solution of Eq. (45) can be written as a power-series expansion in $s$

$$
\tilde{Q}^{(\alpha)}(x, s)=\sum_{n=0}^{\infty} s^{\alpha+n} f_{n}^{(\alpha)}(x),
$$

where the indicial equation determining the allowed values of $\alpha$ is

$$
H_{0} f_{0}^{(\alpha)}+\alpha \phi^{\prime \prime}(x) f_{0}^{(\alpha)}=0
$$

The general solution may then be written as a linear superposition of these particular solutions:

$$
\tilde{Q}(x, s)=\sum_{\alpha} w_{\alpha} \tilde{Q}^{(\alpha)}(x, s) .
$$

We notice that $\alpha=0$ is always a solution of Eq. (47) with a corresponding $f_{0}^{(0)}$ given by

$$
f_{0}^{(0)}(x)=\frac{\exp (-\beta \phi(x) / 2)}{Z^{\frac{1}{2}}} .
$$

The initial condition Eq. (44) means that $w_{\alpha}=0$ for $\alpha<0$, otherwise $\tilde{Q}$ would diverge at $s=0$. The solution must therefore be of the form

$$
\tilde{Q}(x, s)=\sum_{n=0}^{\infty} s^{n} f_{n}^{(0)}(x)+\sum_{\alpha>0} w_{\alpha} \tilde{Q}^{(\alpha)}(x, s) .
$$

It is easy to see, on reconstructing $\rho(\xi)$ from the above Laplace transform, that it must have the form

$$
\rho(\xi) \propto \frac{1}{\xi^{1+\alpha^{*}}}
$$

for large $\xi$ where $\alpha^{*}$ is the smallest strictly positive solution to the indicial equation with $w_{\alpha^{*}} \neq 0$. If there are no solutions to the indicial equation with $\alpha>0$ then $\tilde{Q}(x, s)$ must be given by

$$
\tilde{Q}(x, s)=\sum_{n=0}^{\infty} s^{n} f_{n}^{(0)}(x)
$$

and is analytic in $s$ and all of the moments of $\xi$ will presumably exist; thus $\rho(\xi)$ will not have a power law tail at large $\xi$. The physical arguments leading to Eq. (30) suggest that Eq. (151) should hold when there is a region where $\phi^{\prime \prime}(x)<0$. In addition the same physical argument and the static fluctuation dissipation relation also show that we should have $\alpha^{*}>1$ for finite $\beta$, otherwise $\xi$ will diverge which means that the variance of $x$ on the right hand side of Eq. (10) diverges, which is clearly not possible for a sufficiently confining potential. We shall now confirm this physical picture mathematically. If we define the operator

$$
A_{0}=\sqrt{T} \frac{\partial}{\partial x}+\frac{1}{2 \sqrt{T}} \phi^{\prime}(x),
$$

then clearly we may write $H_{0}=A_{0}^{\dagger} A_{0}$ which shows that $H_{0}$ is positive semi-definite. If $f$ is a solution to the indicial equation Eq. (47), multiplying by $f$ and integrating over all $x$ gives

$$
\int d x f H_{0} f+\alpha \int d x f^{2} \phi^{\prime \prime}(x)=0
$$

When $\phi^{\prime \prime}(x) \geq 0$ for all $x$, it follows that $\alpha \leq 0$. As expected, no power law behavior is possible in this case.

The indicial equation Eq. (47) may also be written as

$$
-T \frac{\partial^{2}}{\partial x^{2}} f+\left(\frac{(1-2 \alpha)^{2}}{4 T}\left(\phi^{\prime}(x)\right)^{2}-\frac{1-2 \alpha}{2} \phi^{\prime \prime}(x)\right) f+\frac{\alpha(1-\alpha)}{T}\left(\phi^{\prime}(x)\right)^{2} f=0
$$


which is equivalent to

$$
H_{\alpha} f+\frac{\alpha(1-\alpha)}{T}\left(\phi^{\prime}(x)\right)^{2} f=0
$$

where the positive semi-definite $H_{\alpha}$ is given by

$$
H_{\alpha}=A_{\alpha}^{\dagger} A_{\alpha}
$$

with

$$
A_{\alpha}=\sqrt{T} \frac{\partial}{\partial x}+\frac{1-2 \alpha}{2 \sqrt{T}} \phi^{\prime}(x)
$$

This thus yields

$$
\int d x f H_{\alpha} f+\frac{\alpha(1-\alpha)}{T} \int d x f^{2}\left(\phi^{\prime}(x)\right)^{2}=0
$$

which implies that $\alpha(1-\alpha)<0$, thus if $\alpha$ is positive then we must have $\alpha>1$, again confirming the physical reasoning of the Introduction.

We shall now show that if there exists a region where $\phi^{\prime \prime}(x)<0$, then there is a solution to the indicial equation with $\alpha>0$, and from the preceding argument, if such an $\alpha$ exits then $\alpha>1$.

We consider the following eigenvalue equation,

$$
H_{0} \psi_{0}+\alpha \phi^{\prime \prime}(x) \psi_{0}=E_{0}(\alpha) \psi_{0}
$$

where $E_{0}$ denotes the ground state energy and $\psi$ the corresponding ground state wave function. Consider this eigenvalue problem at $\alpha=0$, here we have

$$
\begin{aligned}
\left.\psi_{0}\right|_{\alpha=0} & =\frac{1}{Z^{\frac{1}{2}}} \exp (-\beta \phi(x) / 2) \\
E_{0}(0) & =0 .
\end{aligned}
$$

First order perturbation theory shows us that

$$
\begin{aligned}
\frac{\partial}{\partial \alpha} E_{0}(\alpha) & =\int d x \psi_{0}^{2} \phi^{\prime \prime}(x) \\
& =\frac{1}{Z T} \int d x\left(\phi^{\prime}(x)\right)^{2} \exp (-\beta \phi(x))
\end{aligned}
$$

Thus at $\alpha=0$ we have $\frac{\partial}{\partial \alpha} E_{0}(\alpha)>0$, thus there is a region of $\alpha>0$ where $E_{0}(\alpha)>0$ and there can be no acceptable solution $\alpha$ to the indicial equation in that region.

We shall now use the well known variational formula

$$
E_{0}(\alpha)=\min _{\psi} \int d x\left[T\left(\frac{\partial \psi}{\partial x}\right)^{2}+\left(\frac{1}{4 T}\left(\phi^{\prime}(x)\right)^{2}+\frac{1}{2}(1-2 \alpha) \phi^{\prime \prime}(x)\right) \psi^{2}\right]
$$

where the minimum is taken over all functions such that $\int d x \psi(x)^{2}=1$. Define $g=-\min _{x}\left\{\phi^{\prime \prime}(x)\right\}$ and consider the case where $g>0$ and let $x_{0}$ be a point where this minimum is obtained. Without loss of generality we take $x_{0}=0$. Now consider the trial wave function

$$
\psi^{*}(x)=\left(\frac{c}{2 \pi}\right)^{\frac{1}{4}} \exp \left(-c x^{2} / 4\right)
$$

for $c$ large and positive. Using Eq. (64) we obtain

$$
E_{0}(\alpha) \leq \frac{T c}{4}+\left(\frac{1}{4 T}\left(\phi^{\prime}(0)\right)^{2}-\frac{g}{2}(2 \alpha-1)\right)+O\left(\frac{1}{\sqrt{c}}\right)
$$

Thus if $c \gg 1$ and $\alpha \gg c$ then we have $E_{0}(\alpha)<0$. Assuming the continuity of $E_{0}(\alpha)$, along with the fact that $E(\alpha)$ is positive in a region $(0, l)$ for some $l>0$, we have shown the existence of $\alpha^{*}>0$ such that $E\left(\alpha^{*}\right)=0$. 
Now it remains to be shown that in the case where $\phi(x)$ is concave for some range of $x$, that the coefficient $w_{\alpha^{*}}$ in Eq. (48) is non-zero. We recall the boundary condition $P(x, 0)=0$ which means that for large $s$ that $\tilde{Q}(x, s)$ must decay more quickly than $1 / s$ at large $s$.

The Eq. 45) may be written as

$$
\tilde{Q}(x, s)=-\int d x^{\prime} G\left(x, x^{\prime} ; s\right) s \frac{\partial}{\partial s} \tilde{Q}\left(x^{\prime}, s\right) \phi^{\prime \prime}\left(x^{\prime}\right),
$$

where $G$ is the Green's function obeying

$$
\left(H_{0}+s\right) G\left(x, x^{\prime} ; s\right)=\delta\left(x-x^{\prime}\right) .
$$

For fixed $x$ and $x^{\prime}$ and $s \gg 1$ we have from Eq. 68

$$
G\left(x, x^{\prime} ; s\right) \approx \frac{\delta\left(x-x^{\prime}\right)}{s},
$$

which means that for large $s$

$$
\tilde{Q}(x, s) \approx-\phi^{\prime \prime}(x) \frac{\partial}{\partial s} \tilde{Q}(x, s)
$$

and hence

$$
\tilde{Q}(x, s) \approx H(x) \exp \left(-s / \phi^{\prime \prime}(x)\right)
$$

This means that in particular if there is a point $x$ where $\phi$ is concave then $\tilde{Q}^{(0)}(x, s)$ diverges there and thus the full solution needs to have at least one $w_{\alpha} \neq 0$ as $\tilde{Q}^{(\alpha)}(x, s)$ has the same divergent behavior there as $s \rightarrow \infty$, the coefficients must then be chosen to cancel the divergence. A similar mechanism was identified in simplified discrete versions of the the slave equation of the model discussed in this paper [14].

\section{SPECIFIC EXAMPLES}

\section{A. The Simple Harmonic Oscillator}

The simplest example one can consider is the simple harmonic oscillator with

$$
\phi(x)=\frac{\lambda x^{2}}{2} .
$$

From the theory of the precedent section we know that there should be no power law behavior in $\rho(\xi)$, and although the problem can be explicitly solved it is instructive to work through the mathematics as formulated in Section III.

The indicial equation in this case is

$$
-T \frac{\partial^{2}}{\partial x^{2}} f+\frac{\lambda^{2} x^{2}}{4 T} f=\frac{\lambda}{2}(1-2 \alpha) f .
$$

The left hand side of Eq. (73) is the Hamiltonian for a quantum simple harmonic oscillator of mass $m=1 / 2 T$ and frequency $\omega=\lambda$. The energy levels are thus $E_{n}=\left(n+\frac{1}{2}\right) \lambda$ and comparing with the right hand side of Eq. (73) immediately yields that the solutions for $\alpha$ are $\alpha=-n$ and hence, as predicted, are all negative. Clearly the slave equation reads

$$
\dot{\xi}_{t}=-\lambda \xi+1
$$

and so the equilibrium distribution of $\xi$ is a delta function at the fixed point $\xi=1 / \lambda$.

$$
\rho(\xi)=\delta\left(\xi-\frac{1}{\lambda}\right)
$$




\section{B. The Potential $\phi=|x|$}

We now consider the potential

$$
\phi(x)=|x| .
$$

Again there can be no power law behavior. The interesting point about this potential is that the full distribution of $\xi$ can be computed. One can easily solve for $\tilde{P}(x, s)$ to obtain

$$
\tilde{P}(x, s)=\frac{\lambda(s)}{2} \exp (-\lambda(s)|x|) \exp \left(-2\left(\beta s+\frac{1}{4}\right)^{\frac{1}{2}}\right),
$$

where

$$
\lambda(s)=\frac{\beta+\left(\beta^{2}+4 \beta s\right)^{\frac{1}{2}}}{2} .
$$

Then after integrating over $x$, the Laplace transform can be inverted to yield

$$
\rho(\xi)=\frac{\beta e}{\pi^{\frac{1}{2}}(\beta \xi)^{\frac{3}{2}}} \exp \left(-\frac{\beta \xi}{4}-\frac{1}{\beta \xi}\right) \text {. }
$$

Notice that the large $s$ behavior is not that predicted by Eq. (71) due to the delta function singularity in $\phi^{\prime \prime}(x)$ at $x=0$, where this occurs it is easy to see that one has a behavior of the form $\exp (-A \sqrt{s})$ but the conclusions stay the same. Indeed the simplest cases exhibiting a power law distribution in $\xi$ are those where $\phi^{\prime \prime}(x)$ is composed of delta functions, as is the case for continuous piecewise linear or quadratic potentials $\phi$.

\section{The piece-wise continuous quadratic potential}

Here we consider the potential

$$
\phi(x)=\frac{1}{2}(|x|-1)^{2}-h x .
$$

In this case we again expect a power law tail in the distribution of $\xi$. The indicial equation in this case is

$$
\begin{aligned}
& -T \frac{\partial^{2}}{\partial x^{2}} f+\left(\frac{1}{4 T}(x-h-1)^{2}-\frac{1}{2}(1-2 \alpha)\right) f=0 ; x>0 \\
& -T \frac{\partial^{2}}{\partial x^{2}} f+\left(\frac{1}{4 T}(x-h+1)^{2}-\frac{1}{2}(1-2 \alpha)\right) f=0 ; x<0
\end{aligned}
$$

along with the continuity of $f$ at $x=0$ and the jump condition.

$$
-T\left(\left.\frac{\partial f}{\partial x}\right|_{0^{+}}-\left.\frac{\partial f}{\partial x}\right|_{0^{-}}\right)+(1-2 \alpha) f(0)=0
$$

The solution which decays as $|x| \rightarrow \infty$ is

$$
\begin{aligned}
& f(x)=A_{+} D_{-\alpha}(\sqrt{\beta}(x-h-1)) ; x \geq 0 \\
& f(x)=A_{-} D_{-\alpha}(\sqrt{\beta}(h-1-x)) ; x \leq 0,
\end{aligned}
$$

where $D_{p}$ denotes a parabolic cylinder function of index $p$ [15]. Using the jump condition and continuity at $x=0$, along with the identity [15]

$$
D_{p}^{\prime}(z)-\frac{z}{2} D_{p}(z)+D_{p+1}(z)=0
$$

we find that $\alpha$ obeys the equation

$$
\Gamma(\alpha, \beta, h)=0
$$




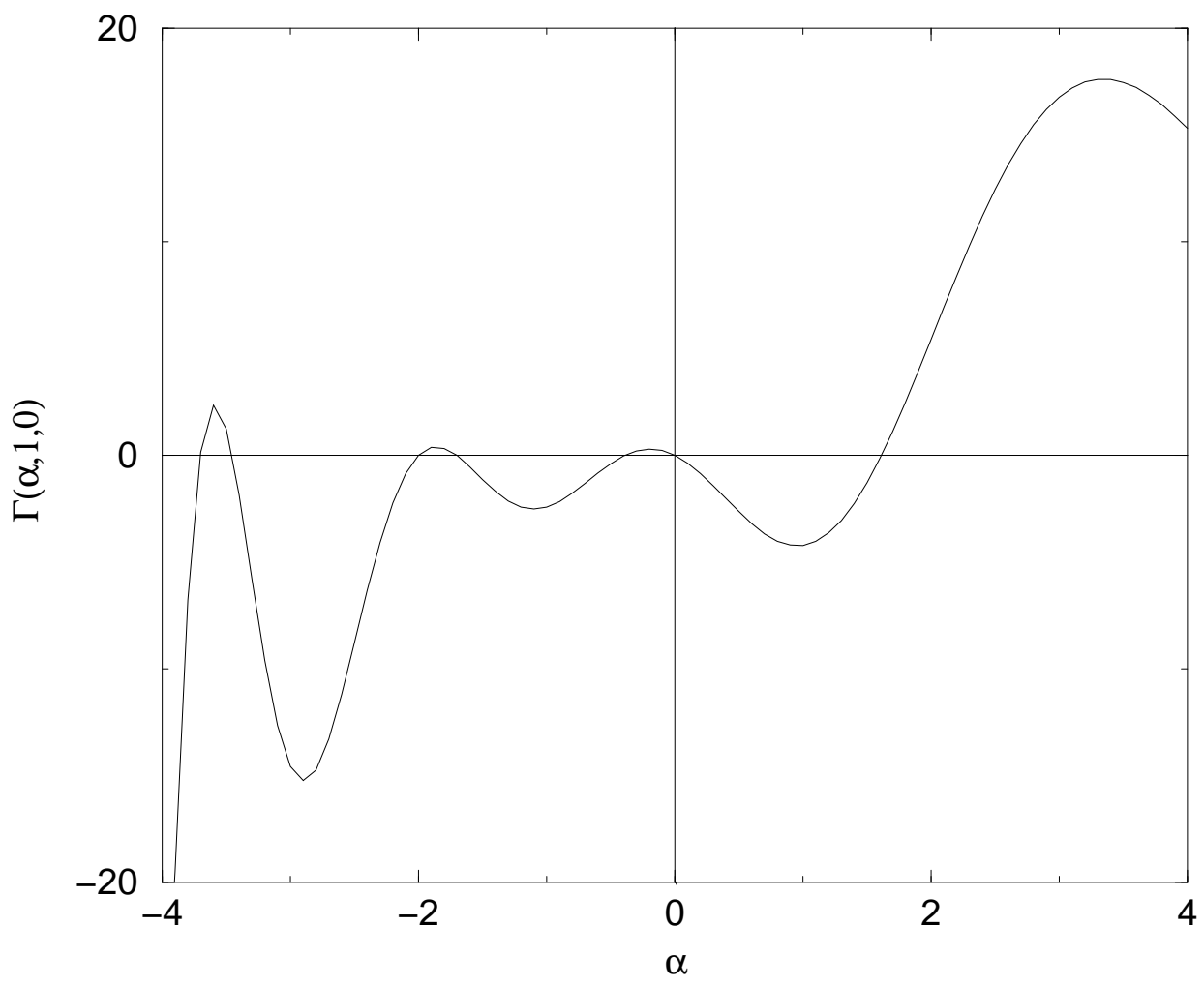

FIG. 2: Graph of $\Gamma(\alpha, \beta, h)$ against $\alpha$ at $\beta=1$ and $h=0$. Note there is only one strictly positive solution to $\Gamma(\alpha, \beta, h)=0$.

where

$$
\begin{aligned}
\Gamma(\alpha, \beta, h) & =2 \sqrt{\beta}(\alpha-1) D_{-\alpha}(-\sqrt{\beta}(1+h)) D_{-\alpha}(-\sqrt{\beta}(1-h)) \\
& -D_{1-\alpha}(-\sqrt{\beta}(1-h)) D_{-\alpha}(-\sqrt{\beta}(1+h))-D_{1-\alpha}(-\sqrt{\beta}(1+h)) D_{-\alpha}(-\sqrt{\beta}(1-h)) .
\end{aligned}
$$

Numerically solving Eq. (85) shows that we have one positive root $\alpha^{*}$, the root $\alpha=0$ while all the others are negative. An example is shown in Fig (2)

At small $\beta$ we can show that

$$
\alpha^{*} \approx 1 / \beta,
$$

while the large $\beta$ behavior depends on the value of $h$. In the case $|h|<1$ the system has two local minima and we find that as $T \rightarrow 0$ then $\alpha^{*}$ is given by

$$
\alpha^{*} \approx 1+\frac{1}{\sqrt{8 \pi \beta}}\left[\exp \left(-\beta(1+h)^{2} / 2\right)+\exp \left(-\beta(1-h)^{2} / 2\right)\right] .
$$

Here we see that in the zero temperature limit $\alpha^{*} \rightarrow 1$. In addition the asymptotic form for $\alpha^{*}$ Eq. 888 tells us that the coefficient $C$ of the power law tail must behave as

$$
C \sim \sqrt{\frac{\beta}{2 \pi}} \exp (-\beta / 2)
$$

in the case when $h=0$. This follows from the static fluctuation dissipation theorem and the fact that the variance of $x$ is non-zero as $T \rightarrow 0$ when $h=0$. Thus although the power law exponent $\alpha^{*}$ decreases, the coefficient of the power law component of the PDF is, in this case, tending to zero exponentially quickly. This can be understood physically as the excursions into the region where $\phi$ is concave are into regions of high energy, whose Boltzmann weight is exponentially suppressed.

In the case $|h|>1$ there is only one local minimum and we find as $T \rightarrow 0$ that

$$
\alpha^{*} \approx 1+\frac{|h|-1}{2} .
$$




\begin{tabular}{|c|c|c|}
\hline$T$ & $\alpha^{*}$ (numerics) & $\alpha^{*}$ (predicted) \\
\hline 2.0 & $2.54(1)$ & 2.560 \\
\hline 1.0 & $1.61(4)$ & 1.612 \\
\hline 0.5 & $1.22(1)$ & 1.191 \\
\hline 0.4 & $1.12(1)$ & 1.121 \\
\hline
\end{tabular}

TABLE II: Exponent $\alpha^{*}$ evaluated from histogram of $\xi$ numerically generated for the potential $\phi$ of Eq. (80) regulated at the origin with $\epsilon=0.05$ compared with analytical prediction of Eq. 855 for various values of $T$ and at $h=0$.

The predictions obtained by our method may be confirmed by numerical simulations. In the numerical simulations one needs $\phi^{\prime}$ to be continuous and so the cusp in the potential at $x=0$ needs to be regularized. We take a small interval $[\epsilon, \epsilon]$ where we set $\phi^{\prime}(x)=(\epsilon-1) x / \epsilon+h$, this choice ensures $\phi^{\prime}(x)$ is continuous and in the limit $\epsilon \rightarrow 0$ we recover the potential of Eq. (80). Simulation results are performed with $\epsilon=0.05$ and compared with the analytical result for the potential $\phi$ of Eq. (80). A table comparing the exponents obtained from the numerical simulation and from solving Eq. (85) for the case $h=0$ is shown in table (III), the agreement is excellent and the deviation is compatible with it being of order $\epsilon=0.05$. Similar agreement is found when $h$ is non-zero.

\section{The 'W' Potential}

Here we consider the 'W' shaped potential given by

$$
\begin{aligned}
\phi(x) & =a|x-1| & & |x| \leq 1 \\
& =|x|-1 & & |x| \geq 1 .
\end{aligned}
$$

The second derivative of the potential is thus given by

$$
\phi^{\prime \prime}(x)=-2 a \delta(x)+(1+a) \delta(x-1)+(1+a) \delta(x+1),
$$

and so from the general theory of section III we expect a power law behavior where $a>0$ and $a<-1$. The indicial equation is given by

$$
\begin{aligned}
& -T \frac{\partial^{2}}{\partial x^{2}} f+\frac{a^{2}}{4 T} f=0|x| \leq 1 \\
& -T \frac{\partial^{2}}{\partial x^{2}} f+\frac{1}{4 T} f=0|x| \geq 1,
\end{aligned}
$$

along with the jump conditions

$$
\begin{aligned}
-\left.2 T \frac{\partial}{\partial x} f\right|_{0}+a(1-2 \alpha) f(0) & =0 \\
T\left[\left.\frac{\partial}{\partial x} f\right|_{1^{+}}-\left.\frac{\partial}{\partial x} f\right|_{1^{-}}\right]-\left(\frac{1}{2}-\alpha\right)(1+a) f(1) & =0 .
\end{aligned}
$$

The solution of the indicial equation, which decays as $|x| \rightarrow \infty$ is

$$
\begin{aligned}
f(x) & =A_{+} \exp (\beta a|x| / 2)+A_{-} \exp (-\beta a|x| / 2) & & |x| \leq 1 \\
& =B \exp (-\beta|x| / 2) & & |x| \geq 1 .
\end{aligned}
$$

Continuity at $x=1$ then gives

$$
B \exp (-\beta / 2)=A_{+} \exp (\beta a / 2)+A_{-} \exp (-\beta a / 2),
$$

allowing for the elimination of the variable $B$. The vector

$$
\mathbf{u}=\left(\begin{array}{l}
A_{+} \\
A_{-}
\end{array}\right)
$$


is then determined by $M \mathbf{u}=0$, where

$$
M=\left(\begin{array}{cc}
-\alpha & 1-\alpha \\
\exp (\beta a / 2) \alpha(1+a) & \exp (-\beta a / 2)(\alpha(1+a)-a)
\end{array}\right) .
$$

The possible values of the exponent $\alpha$ are then determined by the existence of a solution such that $\mathbf{u} \neq 0$, that is to say $\operatorname{det} M=0$, which yields the solution $\alpha=0$ or

$$
\alpha=\alpha^{*}=1+\frac{1}{(1+a)(\exp (\beta a)-1)} .
$$

We see from Eq. (100) that $\alpha^{*}$ is positive and greater than one in the region where $\phi$ has a concave component as predicted by the general theory. In the region $a>0$ we find that

$$
\begin{aligned}
& \alpha^{*} \approx 1+\frac{1}{a(1+a) \beta} \text { as } \beta \rightarrow 0 \\
& \approx 1+\frac{\exp (-\beta a)}{(1+a)} \text { as } \beta \rightarrow \infty .
\end{aligned}
$$

In the region $a<-1$ we find

$$
\begin{aligned}
\alpha^{*} & \approx 1+\frac{1}{|a||1+a| \beta} \text { as } \beta \rightarrow 0 \\
& \approx 1+\frac{1}{|1+a|} \text { as } \beta \rightarrow \infty .
\end{aligned}
$$

We see in the case $a<-1$ in the limit $T \rightarrow 0$ that $\alpha^{*}>1$, this must be the case from the static fluctuation dissipation relation: here there is only one minimum and we therefore have

$$
\beta\left\langle x^{2}\right\rangle_{E} \approx \frac{\beta \int d x x^{2} \exp (-\beta|a||x|)}{\int d x \exp (-\beta|a||x|)} \approx \frac{2 T}{a^{2}},
$$

and hence $\langle\xi\rangle_{E}$ has no divergence as $T \rightarrow 0$.

An interesting point emerges here. If the variance of $x$ is non-zero in the limit $T \rightarrow 0$ the static fluctuation dissipation theorem tells us that the coefficient $\alpha^{*} \rightarrow 1$ in this limit. However, even if the variance of $x$ tends to zero then $\alpha^{*}$ may still tend to one in the zero temperature limit. This is seen in the case of the potential $\phi(x)=(|x|-1)^{2} / 2-h x$ where the minima are only degenerate at $h=0$. The average value of $\xi$ stays finite because the prefactor, denoted in this paper by $C$, of the power law component of the PDF of $\xi$ is tending to zero sufficiently rapidly. In the cases we have examined here it seems that $\alpha^{*} \rightarrow 1$ in the zero temperature limit when there are at least two local minima of the potential $\phi$. It seems possible therefore that the zero temperature behavior of the exponent $\alpha^{*}$ encodes geometrical or topological properties of the potential $\phi$.

\section{CONCLUSIONS}

We have analyzed the equilibrium distribution of a slave variable $\xi_{t}$ which is the estimator of the susceptibility of a one dimensional Langevin process $x_{t}$. Even though the equilibrium statistics of $x_{t}$ are such that all moments are finite (for a sufficiently confining potential), the probability density function of the slave $\xi_{t}$ can have power law tails characterized by a temperature dependent exponent. This power law behavior is present when the potential $\phi$ has a concave component. The origin of this power law can be understood from simple qualitative arguments. The behavior of the power-law exponent can be analyzed in the steady state using the Fokker-Plank equation for the equilibrium joint probability density function $P(x, \xi)$ for $\left(x_{t}, \xi_{t}\right)$. The exponent is large at high temperatures and decreases on decreasing the temperature. As the temperature is reduced its higher order moments of the slave $\xi_{t}$ diverge and ultimately the variance of the slave may diverge thus rendering it a poor estimator for the susceptibility. This pathology in the slave statistics had been observed in Langevin simulations of spin and quantum systems [2, 3]. A number of exactly soluble cases were analyzed and the results confirmed by numerical simulation.

In future work it would be interesting to generalize our results to higher dimensional systems, notably interacting systems where phase transitions may occur. The temporal evolution of the PDF of the slave is also worthy of future study. It would be interesting to know how quickly the tails of the slave's PDF fill out and after what time it becomes 
equilibrated. One would also like to understand over which timescale temporal averages need to be carried out in order to numerically verify the static fluctuation dissipation relation. Finally the analysis developed here could prove useful in the analysis of similar slave variables occurring in Langevin systems.

[1] G. Parisi and Y.S. Wu, Sci. Sinica 24483 (1981)

[2] I.T. Drummond, S. Duane and R.R. Horgan, Nucl. Phys. B 280, 119 (1987)

[3] S.M. Catherall, I.T. Drummond and R.R Horgan, Phys. Lett. B 254, 177 (1991); Phys. Lett. B 284, 351 (1992)

[4] C. Chatelain, J. Phys. A. 3610739 (2003); F. Ricci-Tersenghi, Phys. Rev. E 68, 065104(R) (2003); E. Lippiello, F. Corberi and M. Zannetti cond-mat/0407523 (2004)

[5] L.F. Cugliandolo and J. Kurchan, Phys. Rev. Lett. 71, 173 (1993); J. Phys. A. 27, 5749 (1994)

[6] S.B. Pope, Int. J. Eng. Sci. 26, 445 (1988)

[7] I.T. Drummond and W. Münch, J. Fluid Mech. 225,529 (1991)

[8] I.T. Drummond, J. Fluid Mech. 252,529 (1993)

[9] A. Schekochihin, S. Cowley and J. Maron, Phys. Rev. E 65, 016305 (2001)

[10] S. Tanase-Nicola and J. Kurchan, J. Phys. A. 36, 10299 (2003)

[11] L.F. Cugliandolo, J. Kurchan and L. Peliti, Phys. Rev. E 55, 3898 (1997)

[12] I.T. Drummond, S. Duane and R.R. Horgan, Nucl. Phys. B 220, 119 (1983)

[13] H. Risken, The Fokker-Planck Equation: Methods of Solutions and Applications, (Springer-Verlag Berlin and Heidelberg) (1996)

[14] I.T. Drummond, J. Math. Phys. 25, 2273 (1992)

[15] I.S. Gradshteyn, I.M. Ryzhik and A. Jeffrey, Table of Integrals, Series, and Products, (Academic Press, London) (1994) 